http://dx.doi.org/10.4314/njt.v38i1.19

\title{
A UNIFIED HOSPITAL REFERRAL SYSTEM (UHRS): A CASE STUDY OF NIGERIA
}

\author{
F. I. Amadin ${ }^{1, *}$ and N. Ukeme ${ }^{2}$

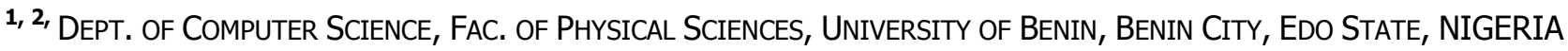 \\ Email addresses: ${ }^{1}$ frankamadin@uniben.edu, ${ }^{2}$ nseukeme@uniben.edu
}

\begin{abstract}
Health care delivery is the duty of every hospital, while some hospitals are equipped with medical experts and cutting edge equipment some are not, this lack of experts and equipment in one hospital calls for the need of the referral of patient from one hospital to another. Base on the vast number of hospitals scattered around the country, locating and having access to these facilities in time remains a problem. Manual referral process is currently adopted by the hospital in finding and referral of patient to a higher facility for treatment, this takes a longer time for the paper process to be completed before actual referral is carried out, hence the need for a Unified hospital referral system (UHRS) in Nigeria. This paper proposes a UHRS system that will facilitate prompt referral.
\end{abstract}

Keywords: - Hospital, Patient, Unified Referral.

\section{INTRODUCTION}

A good state of health of an individual is of great importance to such individual, the state and the nation at large, in this state every activity that is needful ranging from official to non-official, production, business transaction is carried out. When an individual is not in a healthy state, every activity comes to a halt. At this point such individual seeks the attention of a medical professional. Sometimes due to the diverse health condition a particular medical professional may not be able to handle all cases or may not even have the equipment in his facility, when this situation arise there is need to refer such a patient to another medical professional or facility. For successful referral, there must be first and foremost geographical access to referral care facilities. When referral services are accessible, staff must be trained to: provide quality care, offer affordable services, administer essential drugs, provide supplies, and equipment. The most complex aspect of referral care is often the caretaker's acceptance of and compliance with a referral recommendation. This is often determined by a variety of factors such as disease severity, caretaker/community experience, quality of the referral facility, and cost of treatment (time and resources). With the numerous medical facilities in the country choosing the right one at any instance for the transfer of a patient to be made pose a very serious problem. When eventually selected, the referral procedure is started and may take considerable amount of time to finish the process especially if the facility is not located in the same locale as the original facility. The life of a patient is very important, to save these lives it require correct choices and prompt actions to be taken event in the case of an emergency.

\section{BACKGROUND OF STUDY}

The referral of a patient from a basic to a more sophisticated level of health care is considered an integral part of any health care system [6]. Unified hospital referral system (UHRS) has become a necessity in order to create simple and accessible way to support today's medical system.

\subsection{The Nigeria Healthcare System}

[3] The primary objective of the Nigeria healthcare system is to improve the quality, efficiency and effectiveness of healthcare services in Nigeria. The Nigeria healthcare system is provided and maintained by the Federal, State and Local governments, the private sector, as well as religious and non-governmental organizations. The Federal government provides technical support, international relations on health matters, as well as healthcare services to tertiary healthcare system such as the Federal Universities teaching hospitals and the Federal Medical Centers (FMCs). The State government is responsible for secondary healthcare which typically comprises the general hospitals and the state owned University teaching hospitals. The state government provides technical support for secondary healthcare; the Local government is responsible for primary healthcare which is organized through the wards, health districts and health centers [4]. The primary health centers are usually the first point of contact of patients. 
According to [2], "the primary healthcare is supposed to be the first point of contact where patients are then referred to other levels of healthcare". "The referral process is however usually manual, weak and uncoordinated. Consequently, there is information mismanagement in the Nigeria healthcare system". "Hence, knowledge management as well as access to health information is limited. Consequently, data flow is fragmented in vertical silos thereby creating inefficiencies, duplication of efforts, knowledge gap as well as poor evidence based decision" [1].

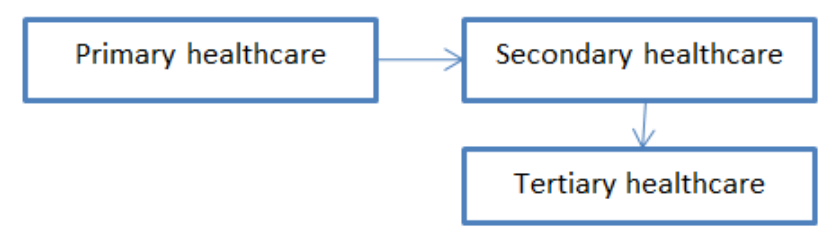

Figure 1: Referral process

\subsection{Information Flow in Nigeria Healthcare System}

According to Strategies for management information flow in Nigerian hospital [7], "when a patient visits a healthcare facility in Nigeria, the first point of contact is usually with a receptionist or an administrative staff who directs the patient to pay for a hospital card". "Once the payment is made, a Medical Record Officer (MRO) attends to the patient by issuing the patient a hospital card and assigning the patient a Hospital ID"." The MRO gets the patient's demographic data such as name, address, sex and records it on the card". "The next point of contact is with a nurse who takes the patient's vital signs and records it on the card". "The nurse then directs the patient to a medical doctor who takes information from the patient regarding his ailment and records it in a case note taking into cognizance the patient's hospital id". "The doctor diagnoses the patients by performing Physical Examination (PE) and recording his findings in the case note and the card". "The doctor may request for a laboratory examination or an imaging procedure based on his findings". "The laboratory technologist takes blood, urine or other sample of the patient and performs test on it while a radiologist takes the image of the patient". "The medical doctor interprets the laboratory or radiologist's results; and either admits, treats or refers the patient based on these results". "During the process of treatment, the doctor prescribes drugs to the patient which the patient gets from a pharmacist". "Patients on admission will eventually be discharged if responded to treatment while those that do not respond well are referred to the next level of care".

\subsection{Levels of Health Care}

There are four levels of health care service in Nigeria, these levels comprises of the various hospitals. Among the various levels of healthcare, Patients' information is not usually delivered promptly to healthcare practitioners at the point of care hence healthcare practitioners do not have immediate and timely access to patients' information. Moreover, information is usually delivered manually to the care providers by the patients hence making the process time consuming.

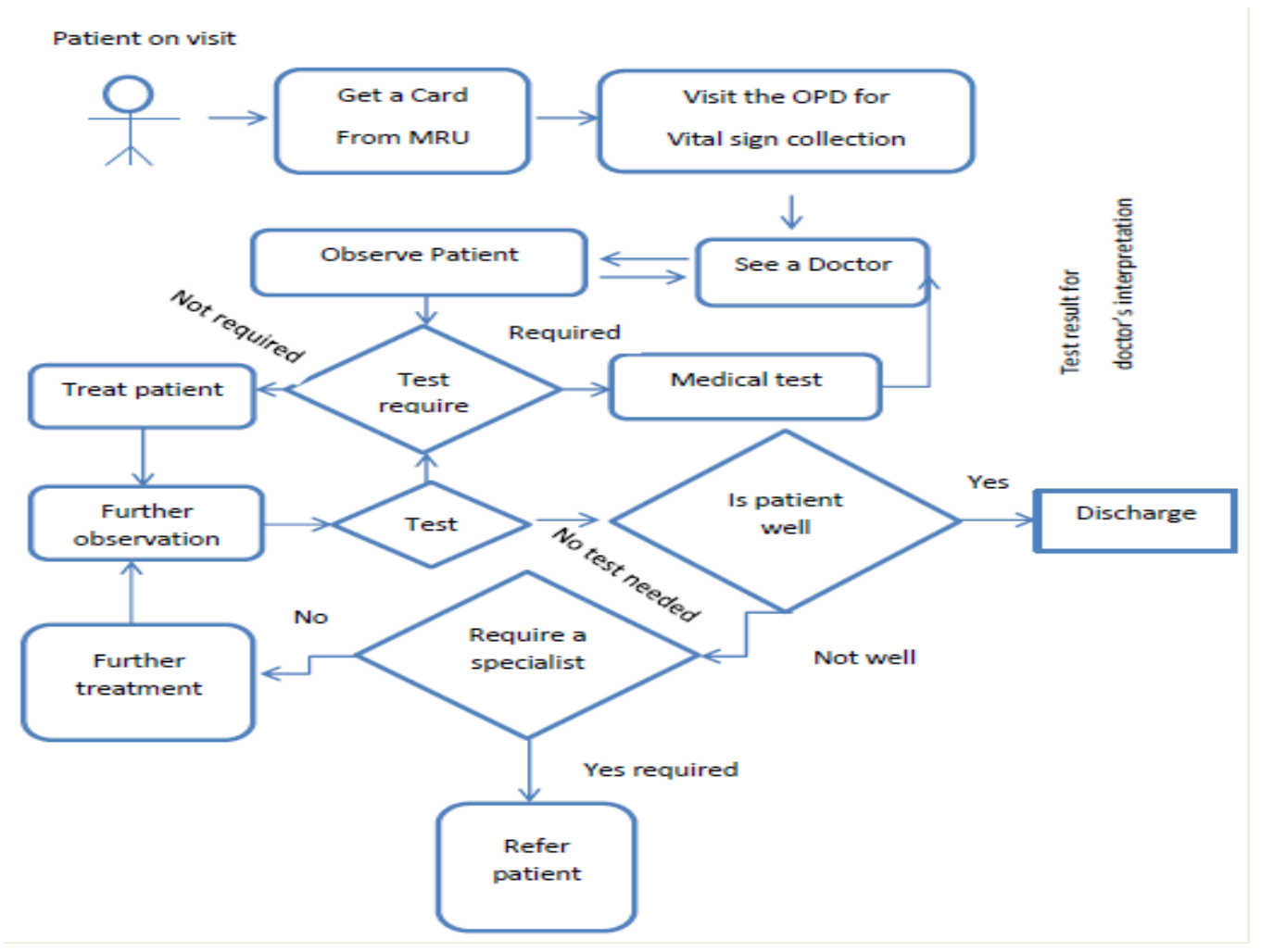

Figure 2: Information flow in the Nigerian healthcare system. 


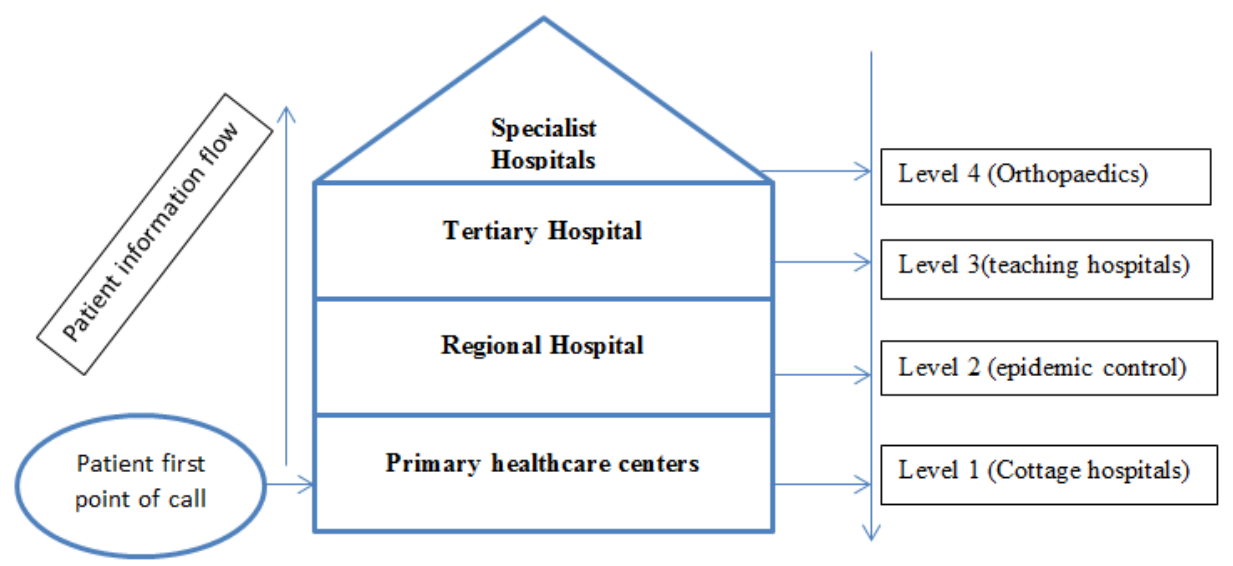

Figure 3: Levels of health care in Nigerian.

Consequently, decisions concerning patients' healthcare is delayed which in turn affects the patient's care adversely. The referral of a patient dose not outrightly leaves the care of patient at the hands of the receiving professional alone but also to lack of health information system integration [5] and poor or no immediate communication mechanism amongst healthcare providers when patients are referred from one healthcare institution to another.

\section{METHOD}

System design focuses on the specification of computer based solution. It is the decomposition of a whole system into component part, studying these parts and providing a computer base solution for the component parts. At this level, this section will be focusing on the technical design of the UHRS.

\subsection{The Propose System}

The new system is designed to remedy these problem posed by the current referral system in figure 4 and 5 , with emphasis on reducing the amount of time spent on patient referral processes, increasing the information dissemination rate there while providing patient with sufficient time to arrive at the referred facility to access care.

\subsection{System Architecture}

The new system architecture eliminate the sequential order of referral that exist in the existing system architecture, its design reduces the time of searching for a hospital to which a patient will be referred. The system architecture provide a point -to-point mode of communication between the referring and receiving hospital,

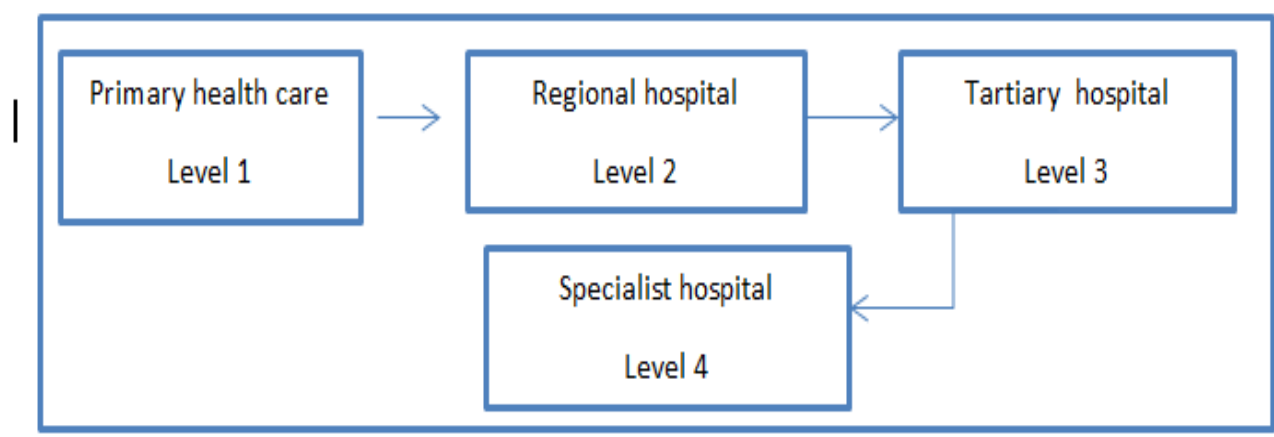

Figure 4: Current referral structure

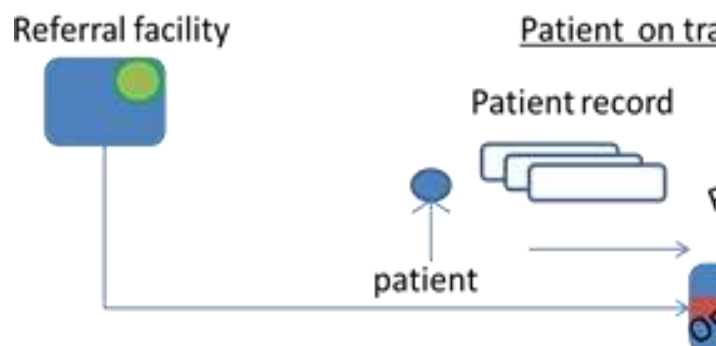

Patient on transit 


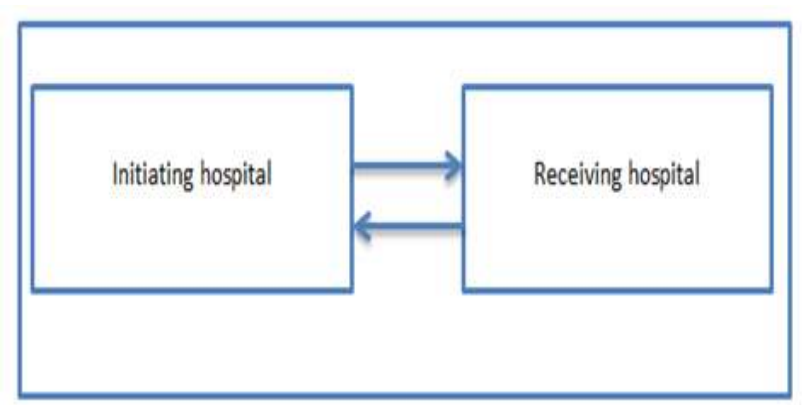

Figure 6: Propose system architecture

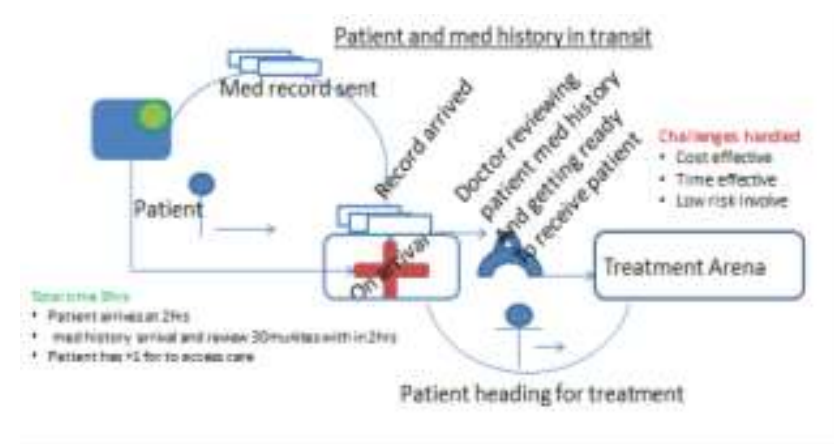

Figure 6b: Patent and medical record in transit

In this design, the patient and the patient medical record are treated differently but in synchronous mode with time, figure $6 \mathrm{~b}$ reveals that while patient is in transit, the medical record is generated and sent via network. The medical expert logs in to study the patient case file, understands the medical history and take medical decision such as the type of standard medical procedure to adopt in treating the patient, drug type and if a team will be needed. On arrival at facility, the patient is moved to the treatment arena without any further delay.

\subsection{Operability of the System}

The system operates in a linear form allowing the hospital user to send an HTTP request to the application server. The request sent is being process and information is been sent back to the user in such a way that the content will be rendered by the web browser.

In line with new system architecture, multiple users (hospitals) are allowed access to the system simultaneously and sharing resource as well information. In Figure 8 the web server handles multiple users sharing information between them.

Communication between the referring hospital and the receiving hospital can be seen in Figure 10.

\subsection{SEQUENCE DIAGRAM.}

A sequence diagram depicts how object interact with each other via messages in the execution of an operation.

\subsection{Uses-Case Diagram}

The use-case diagram is used to present a pictorial view of the key actor in a system and how these actors also interact with the system. This is shown in Figure 13.

\subsection{System Flow Chart}

The system flow chart presents a pictorial representation of the UHRS entire system. Figure 14 shows the system login flow.

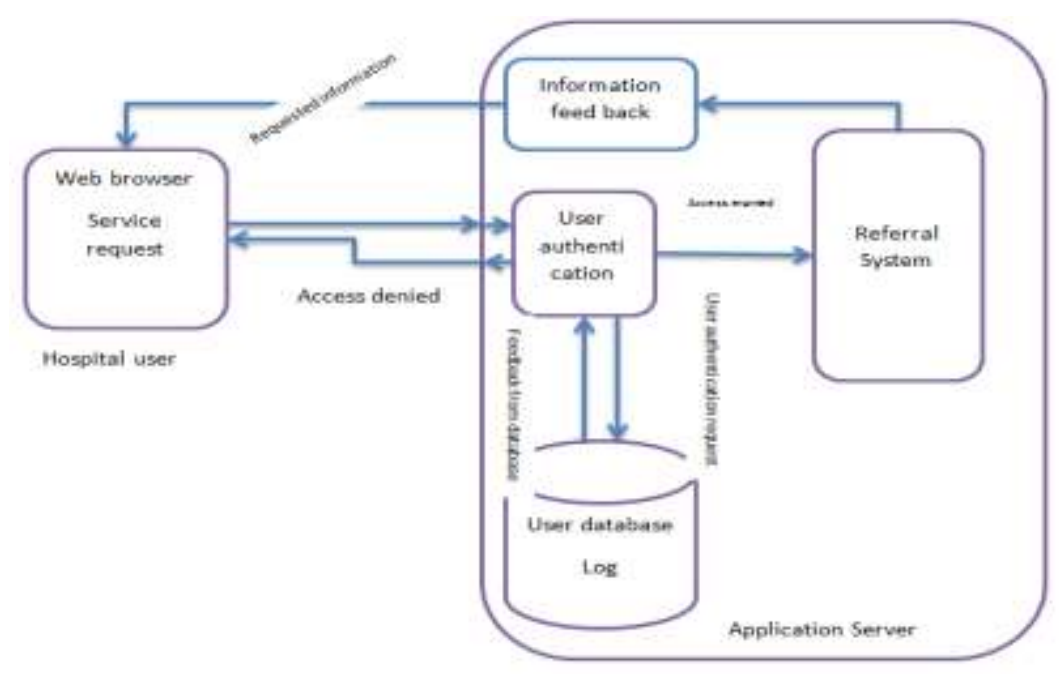

Figure 7: Client/server operation 


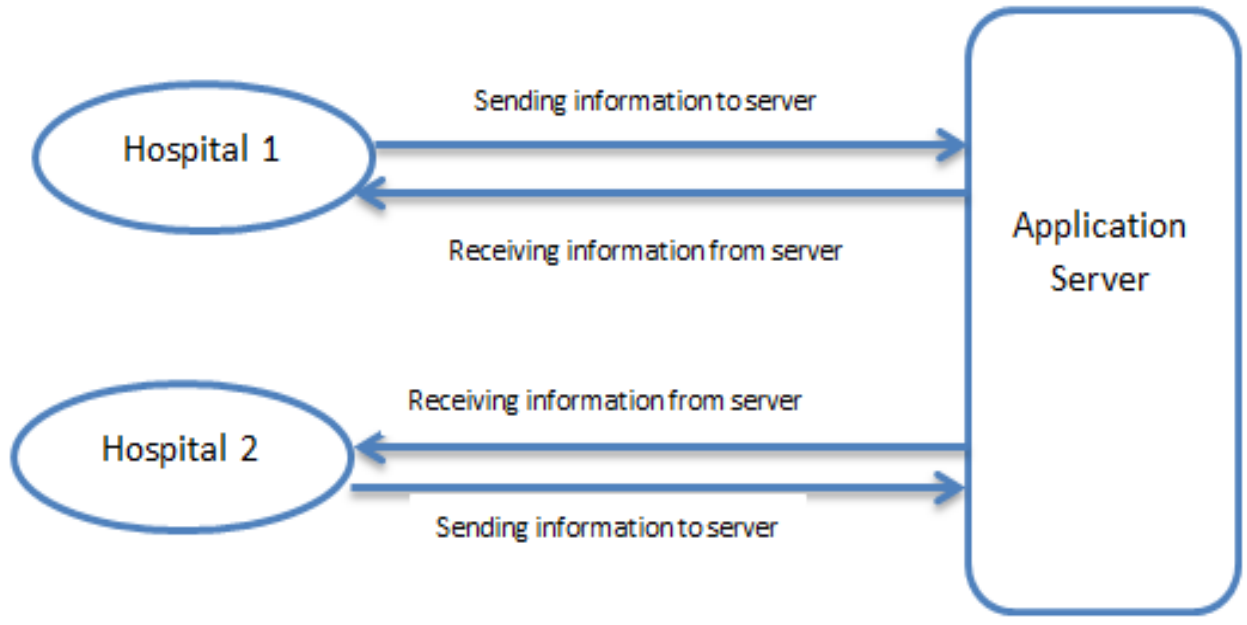

Figure 8: Multiples user connected to the server
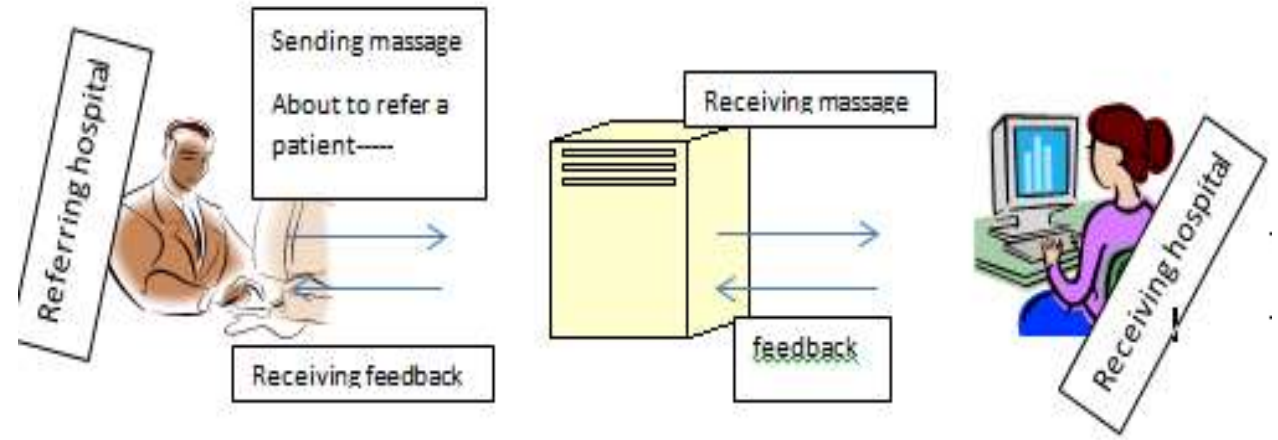

Figure 10: Information flow between the hospitals

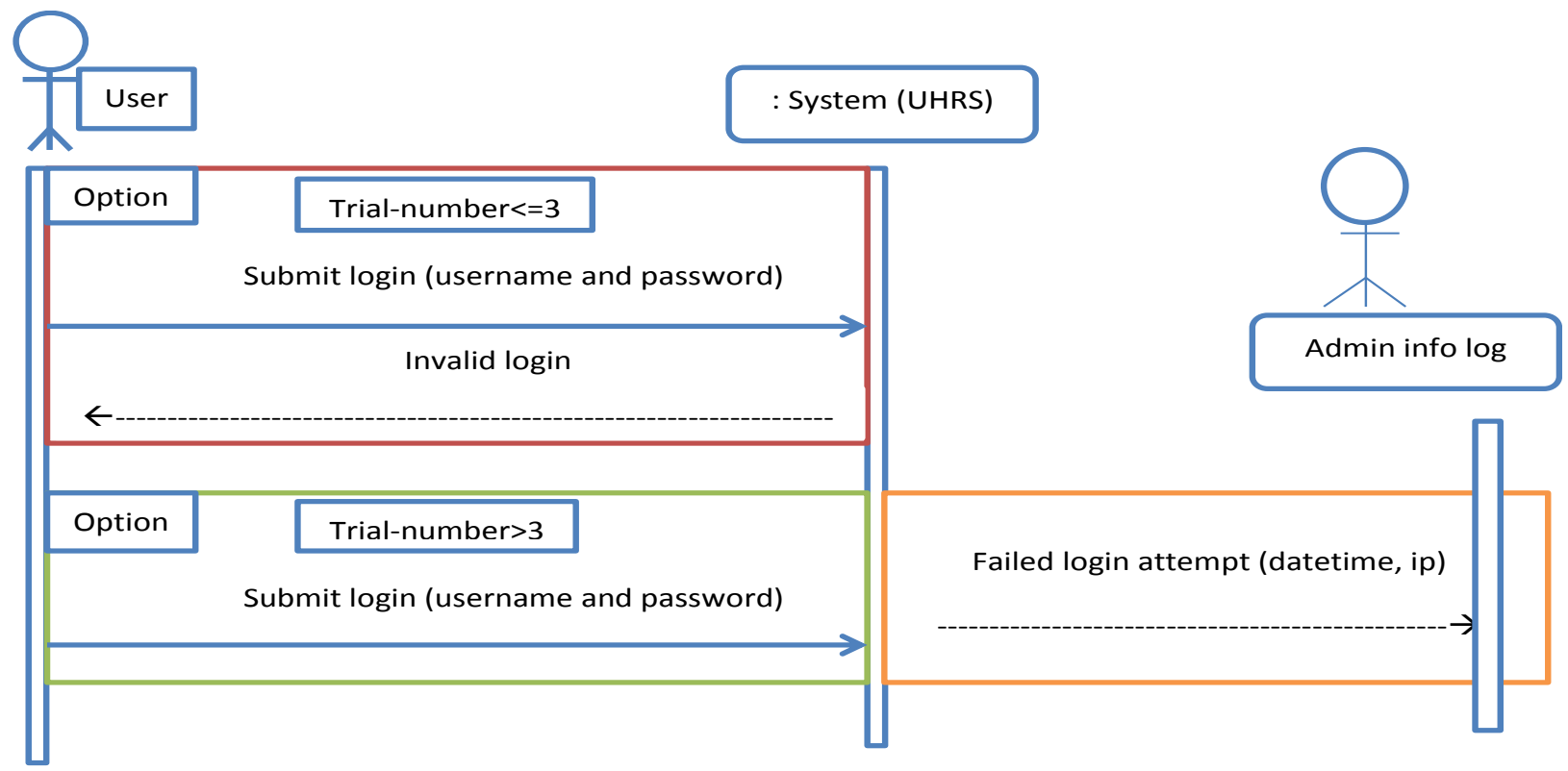

Figure 11: Showing the login sequence diagram 

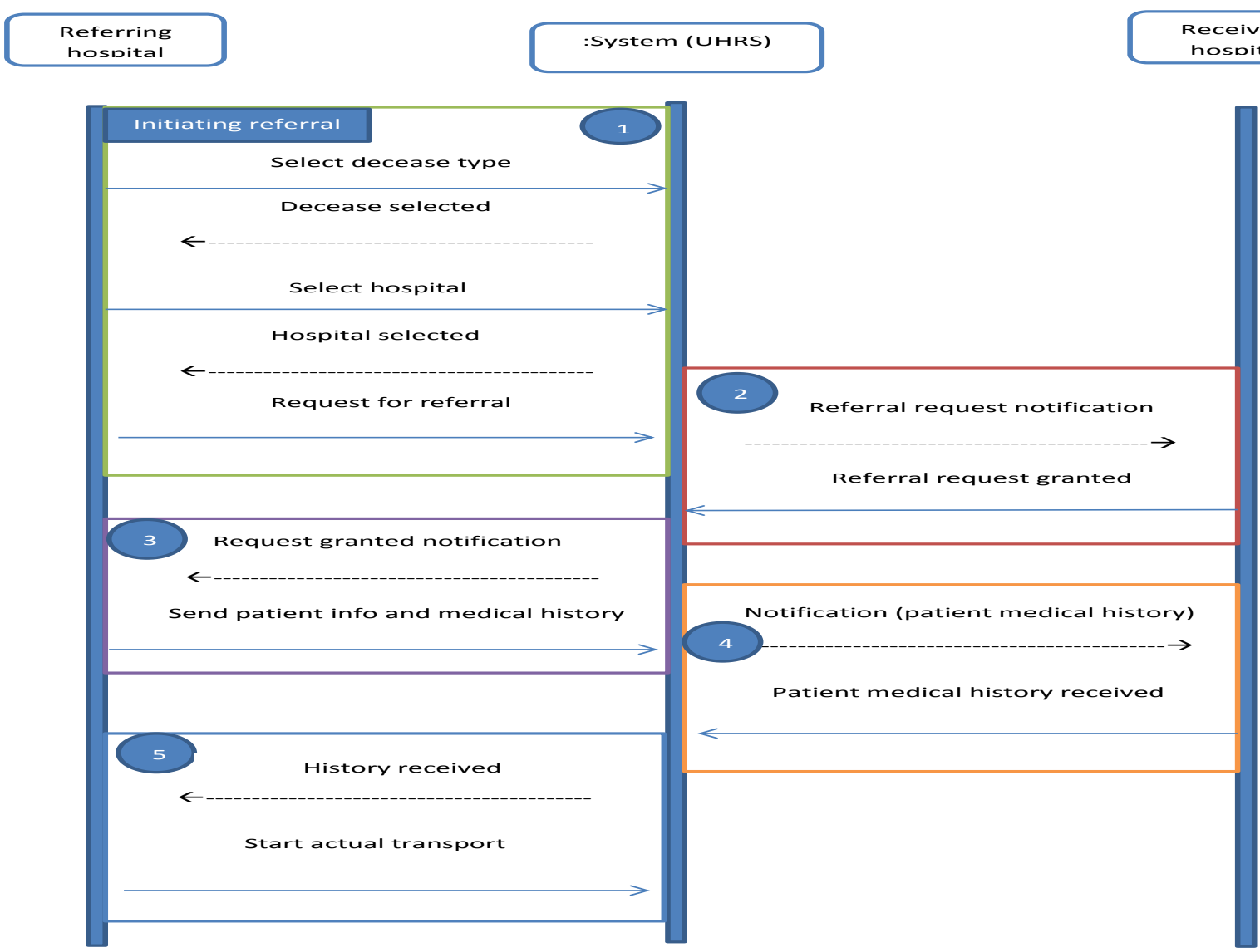

Figure 12: Patient referral sequence

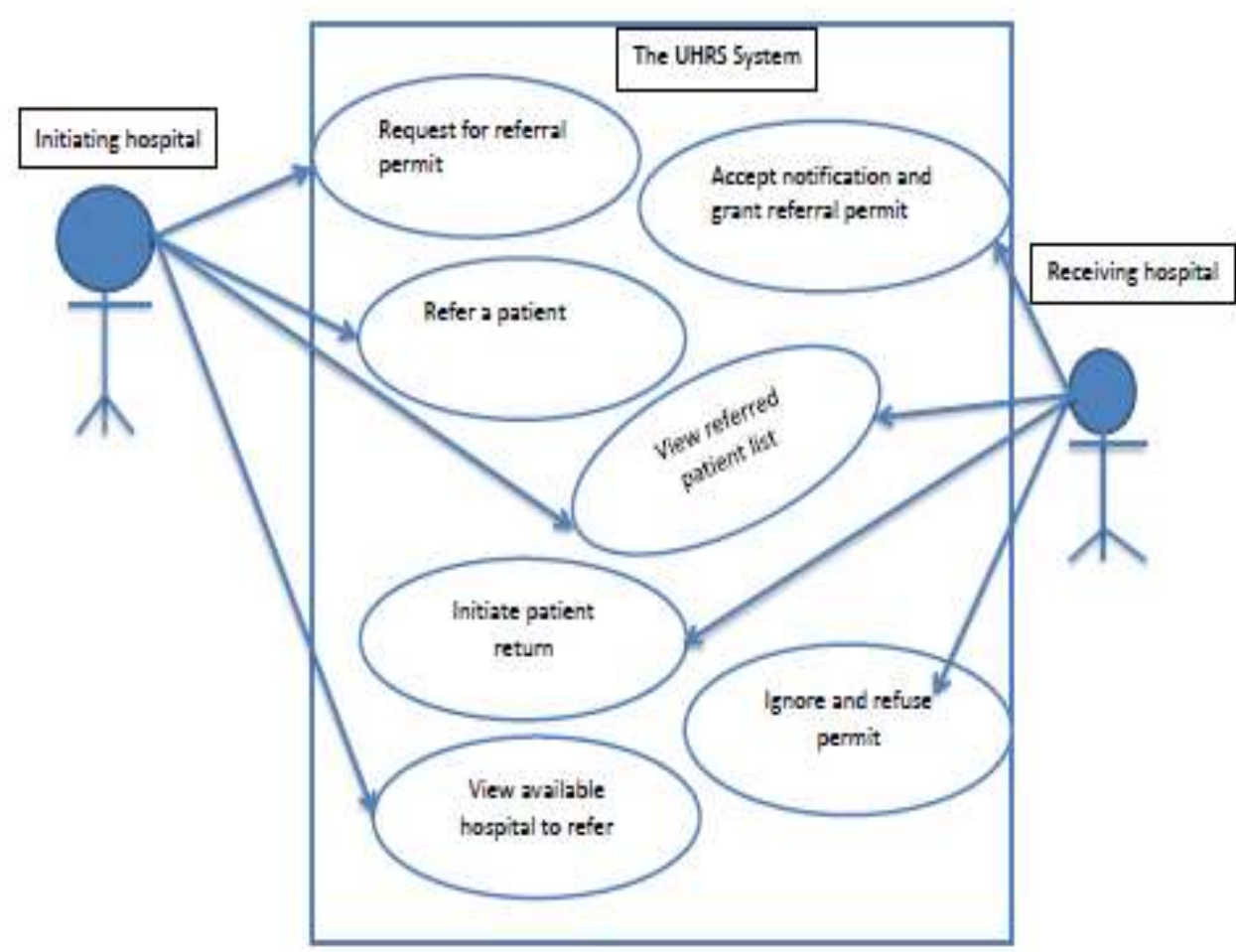

Figure 13: The use-case element of the system 


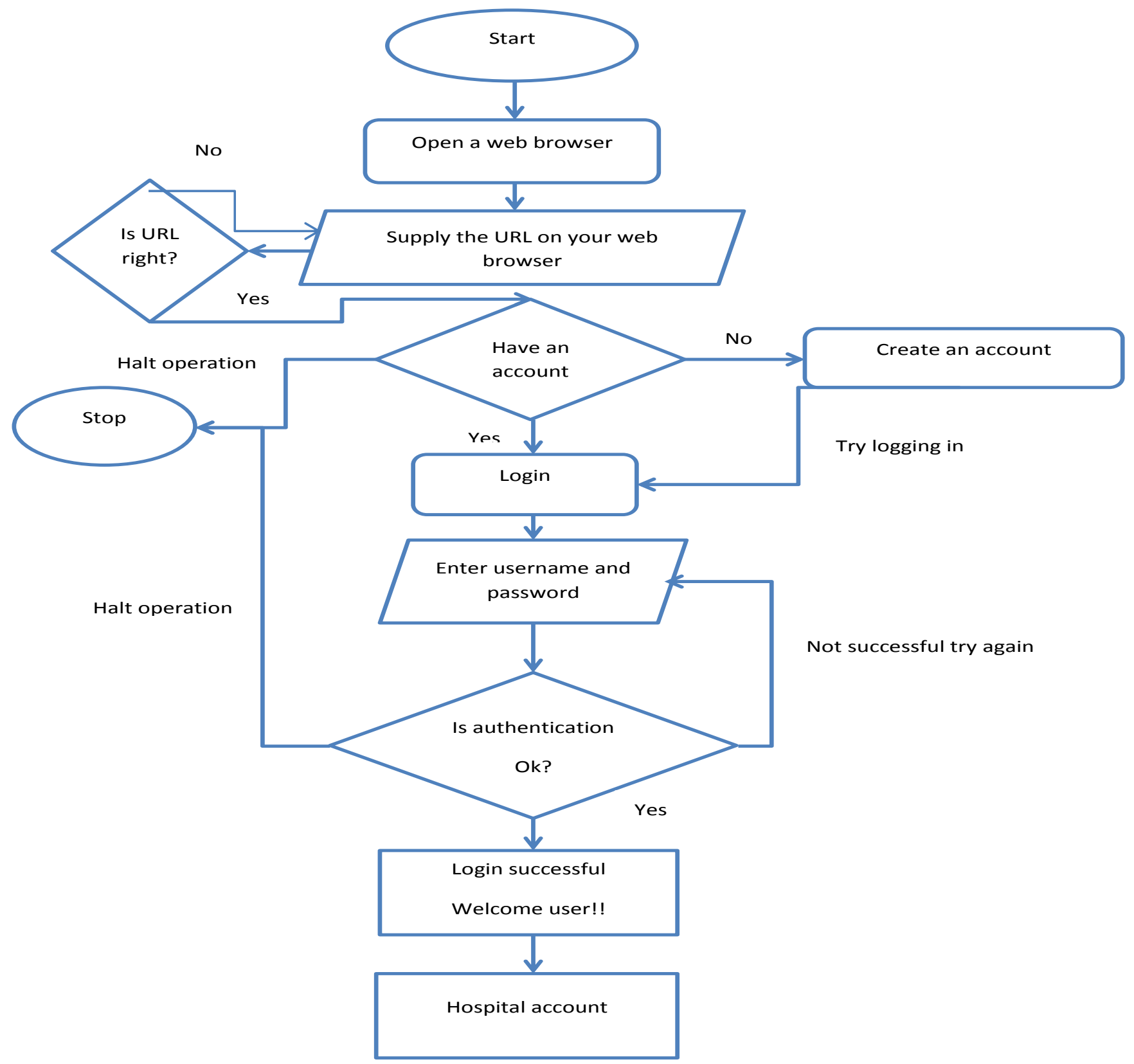

Figure 14: Showing authentication. 


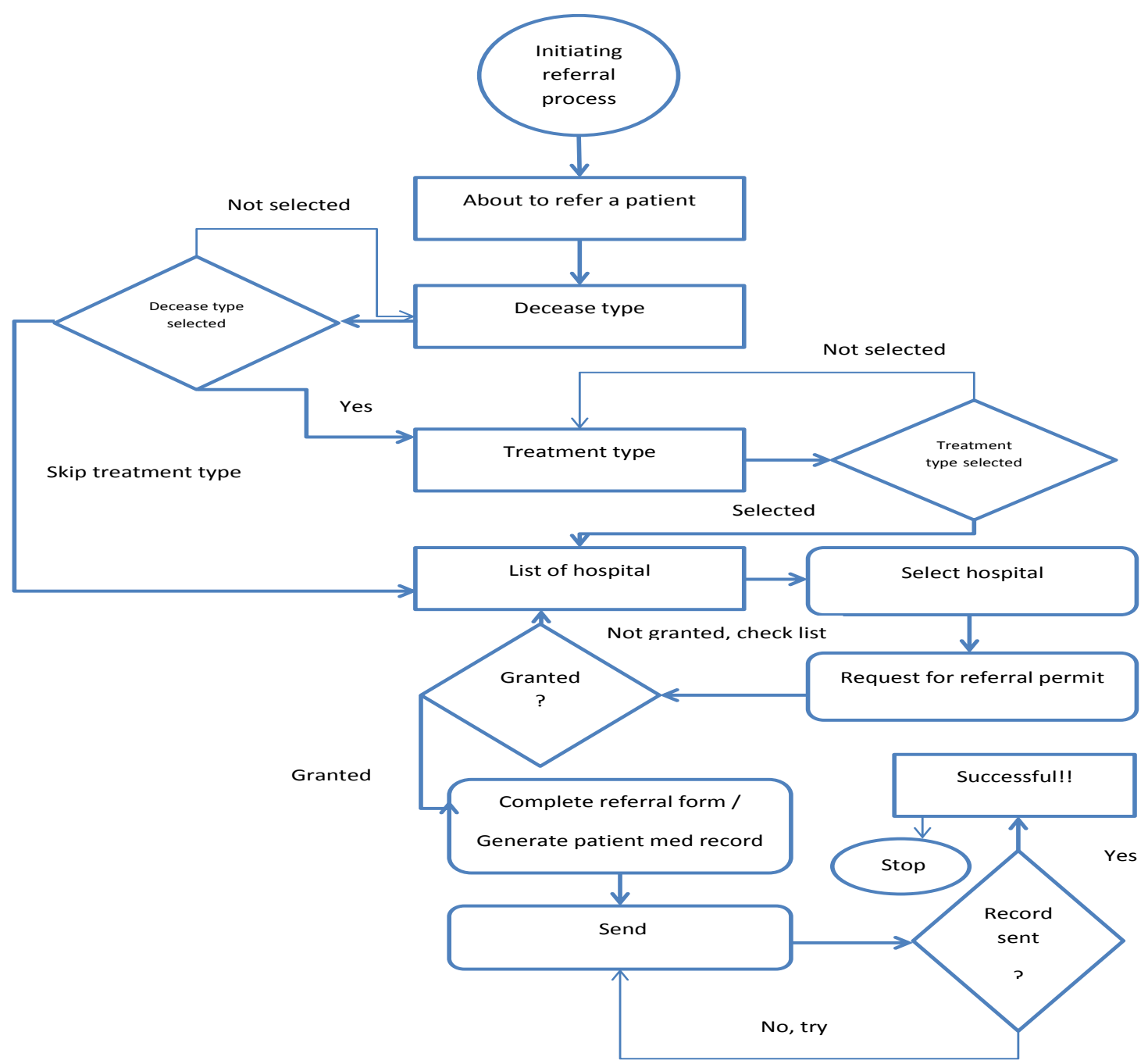

Figure 15: Referral flow

\subsection{Referral Flow Chart}

This chart shows the referral process from start to the end in sequence unlike the manual system this happens in seconds. From the hospital account, initiate referral.

\section{RESULT AND DISCUSSION}

To ascertain the level of acceptability of the proposed system and to understand the current challenges around the referral process faced by personnel in the health care system, a survey was carried out in 10 medical facilities. 20 staff were interviewed using a structured questionnaire consisting of 10 specific questions. The analyzed data, the result revealed a shocking secret. The first five questions had to do with the medical facility and the last five questions were focused around the proposed system.

Figure 16 reveal that the 20 hospitals currently runs automated health care system, 19 admitted that their automated health care system has been effective in handling medical processes within the hospital but ineffective in the referral process, while 16 revealed that the task is daunting and time wasting, 20 staff from each hospital believe that a more effective referral system need to be developed mainly to boost the referral process. Figure 17 specifically reveals the dependent staff contribution to the survey their positive and negative responses.

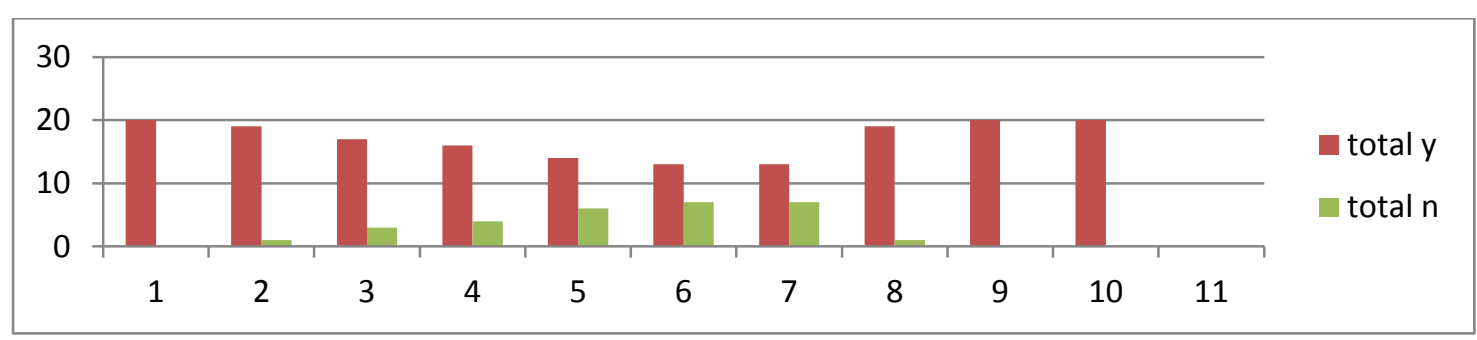

Figure 16: A cross section of staffs in agreement relating to specific questions 


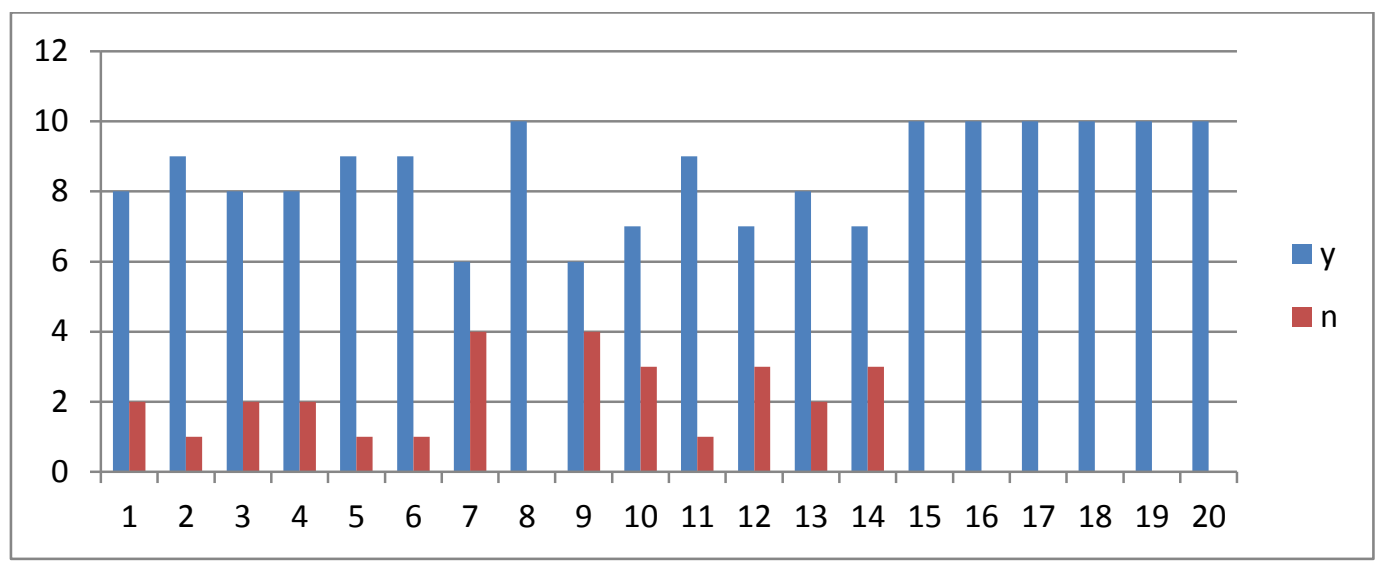

Figure 17: An independent staff contribution to the survey

In other to understand why 200 staffs from 10 medical facility with automated health care system will verge for the UHRS system, the current and proposed system were evaluated based on some specific criteria which are Effectiveness, Timesaving, Accuracy, Ease of referral document processing.

Effectiveness: this criterion ensures that the system will work as initially intended to work without deviation on matter the situation.

Timesaving: time is critical in the health care system, the system must ensure that time is not wasted in the initialization and complete of referral process.

Accuracy: the system ensures an accurate prediction of hospital, such that a patient will need not be re-referred to another medical facility for treatment.

Ease of referral document processing: referral document processing is a very tedious task; the system must ensure that this process is not a daunting task for personnel to carry out from scratch to completion.

\subsection{Evaluation of the current referral system}

The current system evaluation gives a shocking revelation; the system based on the evaluation is inaccurate in the prediction of appropriate hospital, time consuming and uneasy to complete referral process in time. Although effective base on some medical standard operation in some medical facilities, the highest peak of effectiveness it not acceptable and it is still an issue that needs to be addressed.

\subsection{Evaluation of the proposed system}

Many staffs believe that the current system can perform better if joined by a more dedicated referral system. This system will specifically be used for referral processes. With all its criteria scaling above average figure 17, it is believed that the proposed UHRS system will be more effective, time saving, accurate and easy to use to fast track the referral process.

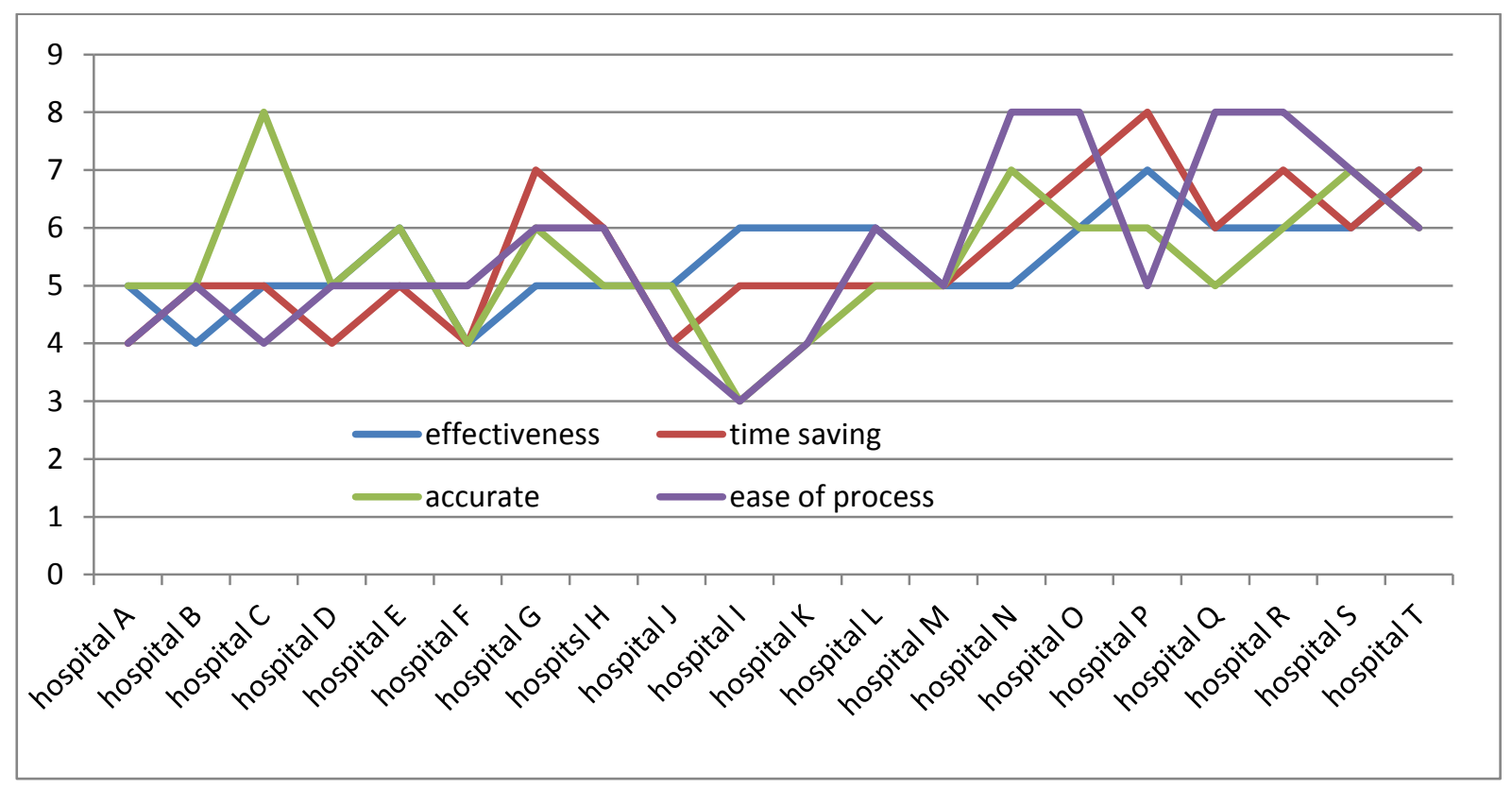

Figure 18: Current system evaluation 


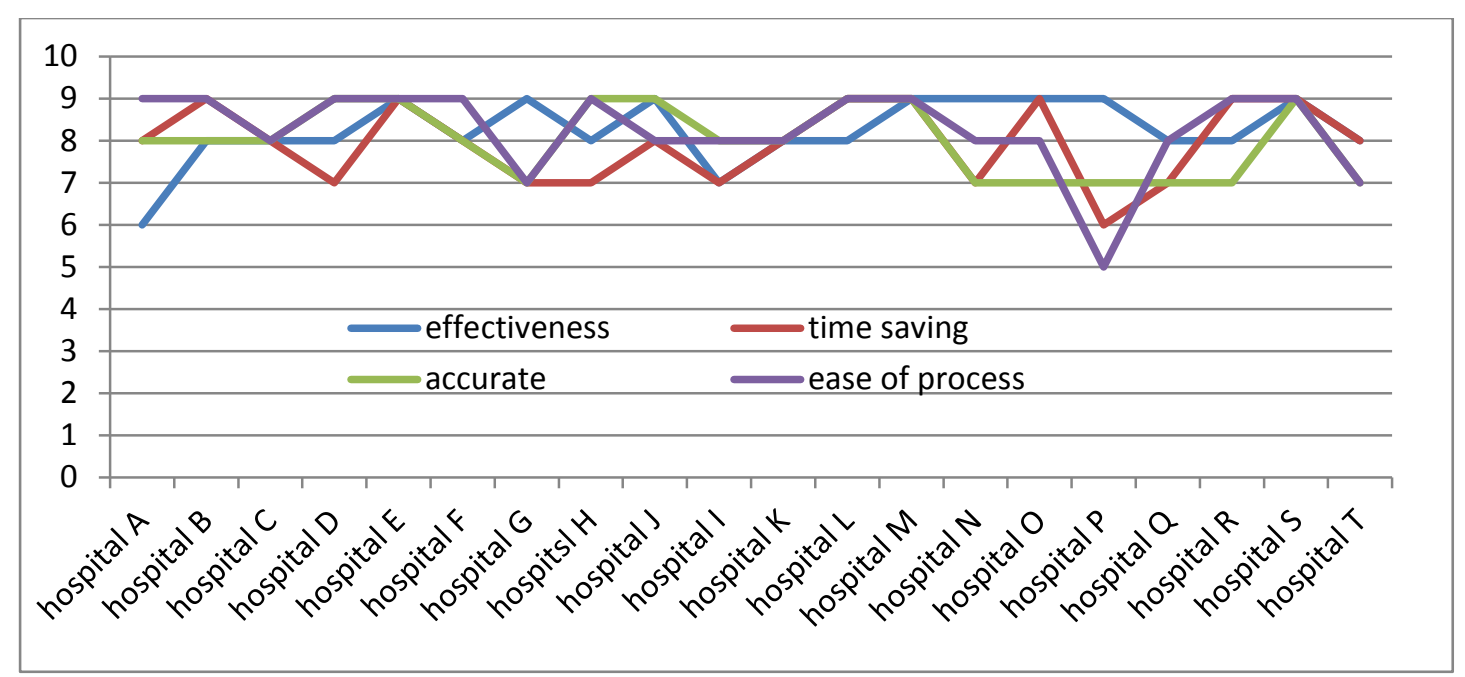

Figure 19: The propose system evaluation

\subsection{System interface design}

System implementation is the construction of the new system and the delivery of that system into production (that is, the day-to-day business or organization operation). The actual transfer of patient will not be carried out without the request for and permission granted from the chosen hospital, the referral request module aid in the making of a fast request just at the click of a button. The interface enables for the easy search of a specific hospital base on the a specific decease. In order to narrow down the search, base on the evaluation of the patient, a specific decease type will be fed into the system, on the click of the search button, the system automatically searches for the hospital and gives a result of the closest and most free hospital, with this result a patient referral request will be made. At the grant of request, actual referral can then be made. In figure 16 , the list for a heart decease treatment hospital is made, and a list of five hospitals are returned with $\mathrm{T}-\mathrm{H}$ Lagos meeting the specific requirement.

The system is design with new features that allows for easy and fast generation of patient health report, though is it allowed for the user to decide on the mode to use. The system provides a manual and automated mode of patient health report generation, figure 17 shows the report generation interfaces while figure 18 and 19 shows the actual report.

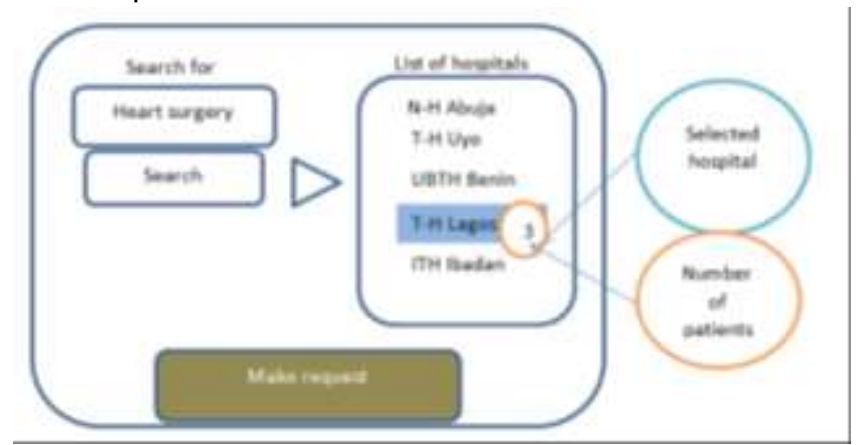

Figure 20: Referral request module showing the observed dataset of hospital that carry out heart surgery

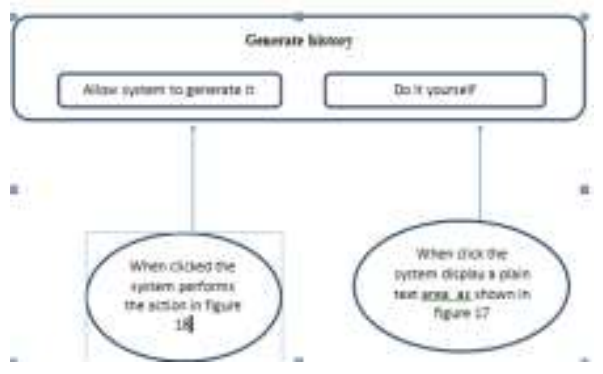

Figure 21: Patient health report generating interface

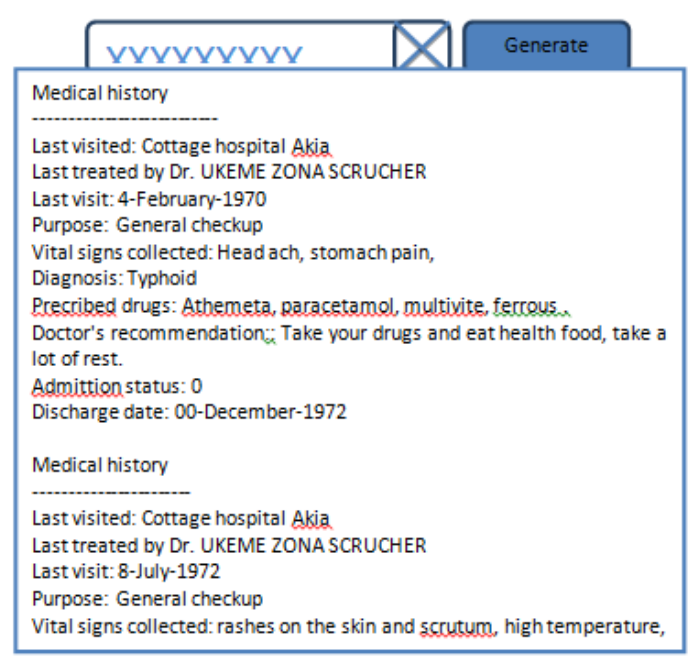

Figure 22: Auto generated patient health report

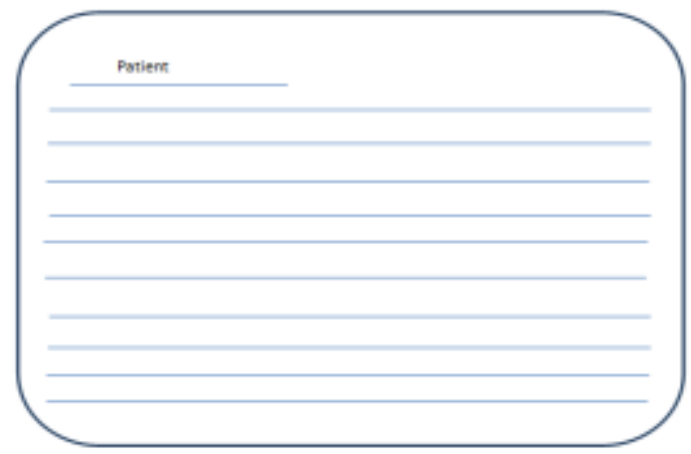

Figure 23: Interface for manual patient health report generation 


\section{CONCLUSION}

The designed unified hospital referral system will be a support system that will help save help lives of many Nigerians especially in the area of ease of referral of patient to a needed health care delivery center.

This paper has taken a critical look into the health delivery system especially in the aspect of referral. In the course of this paper reveals the loop hold in the Nigerian health care system to be wrapped around time and delay of information flow. Time is a critical element as time waiting can cause serious problem or even the death of a patient. To remedy this, UHRS system has been designed.

The UHRS as designed is a three layered application system. This system is designed such that it allow for the ease of communication among the various hospitals in the Nigerian health delivery system.

There exists no doubt that the government of Nigeria may not be able to supply all medical facilities in the country with the needed equipment for adequate operation. But one thing is sure, some of the facilities will be provided with this equipment either as a whole or in fractions. The need to access these facilities and use this equipment is essential as some of the patient may not have direct access to the facility where the equipment are but will need to be referred by other medical facility. To facilitate this referral process at a faster rate the unified hospital referral system (UHRS) is highly recommended as it will help in reducing patients pains by reducing the time taken to initiate and complete referral processes, thereby putting more time on the side of the patient to arrive at the referred medical facility and access care.

\section{REFERENCES}

[1] Annan K. Executive Summary of Strengthening Health Systems in Nigeria. The Nigerian Academy of Science. Lagos, Nigeria. 2002.

[2] Akande T. M Referral System in Nigeria: Study of a Tertiary Health Facility. Annals of African Medicine 3(2004), 130 - 133. 2004.

[3] Iroju O. G. Capacity Building in Electronic Health in Nigeria Healthcare Delivery System. In Proceedings of 3nd Interdisciplinary International Conference, Nigeria. 2014

[4] Iroju O., Soriyan A., Gambo I., \& Olaleke J. Interoperability in Healthcare: Benefits, Challenges and Resolutions. International Journal of Innovative and Applied Studies, Morocco, 3(May. 2013), 262270. 2013.

[5] Mohammed D.. National Health Insurance Scheme: Towards Universal Coverage By 2020. The Nigerian Academy of Science. Lagos, Nigeria. 2002.

[6] Murray, S. \& Pearson S. Maternity referral systems in developing countries: current knowledge and future research needs, Social Science and Medicine, 62 2205-2215, 2006. 2006.

[7] Strategies for management information flow in Nigerian hospital Retrieved from http:// www. oluwaseun-2015-ijais-451443.pdf. 2015. 